\title{
Caspofungin Use in Daily Clinical Practice for Treatment of Invasive Aspergillosis: Results of a Prospective Observational Registry
}

\author{
Johan Maertens ${ }^{1 \dagger}$, Gerlinde Egerer ${ }^{2 \dagger}$, Wan Shik Shin ${ }^{3+}$, Dietmar Reichert ${ }^{4 \dagger}$, Michael Stek ${ }^{5 \dagger}$, Sheenu Chandwani ${ }^{6 \dagger}$, \\ Malathi Shivaprakash ${ }^{7 *+}$, Claudio Viscoli ${ }^{8+}$, the study team CAN-DO(IA)
}

\begin{abstract}
Background: A prospective observational registry assessed real world experience with caspofungin monotherapy or combination therapy for the initial or salvage treatment of proven or probable invasive aspergillosis (IA).

Methods: Data were collected from April 2006 to September 2007 for patients treated with caspofungin for a single episode of IA. Clinical effectiveness was categorized as favorable (complete or partial) or unfavorable (stable disease or failure) at the end of caspofungin therapy (EOCT).

Results: Consecutive patients $(n=103)$ with proven or probable IA (per EORTC/MSG criteria) were identified from 11 countries. Malignancy (76.7\%), neutropenia (64.1\%), allogeneic hematopoietic stem cell transplantation (HSCT, 22.3\%), solid organ transplantation (8.7\%), autologous HSCT (4.9\%), and HIV/AIDS (2.9\%) were the most common underlying conditions. Most patients (84.5\%) had pulmonary IA. Aspergillus fumigatus was the most frequently isolated species. The majority of patients received caspofungin monotherapy (82.5\%) primarily as salvage therapy (82.4\%). The main reason for switching to salvage therapy was clinical failure of the first-line therapy (69\%). A favorable response at EOCT was seen in 56.4\% (57/101) of patients overall, including 56.5\% (48/85) and 56.3\% (9/ 16) of patients receiving caspofungin monotherapy and combination therapy, respectively. Favorable response rates in clinically relevant subgroups were: malignancy, 51.9\% (41/79); allogeneic HSCT, 56.5\% (13/23); and neutropenia at time of hospitalization, 53.0\% (35/66). There was a 72.3\% (73/101) survival at 7 days after EOCT. Serious adverse events related to caspofungin were reported in 4 cases (3.9\%); 3 patients (2.9\%) discontinued treatment due to an adverse event related to caspofungin.
\end{abstract}

Conclusions: Caspofungin was both effective and well tolerated among high-risk patient groups such as those with neutropenia and active malignancies.

\section{Background}

Invasive aspergillosis (IA) accounts for approximately $30 \%$ of all invasive fungal infections [1]. In spite of recent improvements in our ability to diagnose the disease at an earlier stage and the availability of new antifungal drugs, IA still carries a high mortality rate $[2,3]$. In addition, in carefully selected study patients with proven, probable or possible IA, overall response rates are approximately $50 \%$ for both voriconazole $(52.8 \%)$, a current drug of choice, and for liposomal amphotericin $B$

\footnotetext{
* Correspondence: malathi_shivaprakash@merck.com

+ Contributed equally

${ }^{7}$ Merck \& Co., Inc. Whitehouse Station, NJ, USA
}

(46-50\%), the currently recommended alternative for first-line therapy $[1,4,5]$. Response rates are considerably lower among allogeneic hematopoietic stem cell transplant recipients (HSCT) than in patients with active hematologic malignancies $[4,5]$.

Caspofungin is the first echinocandin approved for use in the treatment of IA in patients who are refractory to or intolerant of other agents. In this setting, caspofungin monotherapy has an overall success rate of $45 \%$ to $60 \%$ in the context of clinical trials and outside clinical trials in an open case setting [6-9]. However, there is a need for continuing studies of caspofungin efficacy and tolerability because the efficacy of these agents may change
C Biomed Central 
over time and is likely to vary in different real-world practices compared with clinical trials. This article describes a prospective observational registry developed to assess daily clinical practice with caspofungin when used as monotherapy or in combination therapy for initial or salvage treatment of proven or probable IA.

\section{Methods}

A prospective observational registry was developed to collect data from consecutive patients treated with caspofungin monotherapy or caspofungin combination therapy for proven or probable IA. The protocol was approved by the institutional review boards at each participating center. Data collection was conducted in accordance with the ethical standards outlined in the declaration Helsinki and guidelines for good clinical practices [10].

\section{Patient Population}

Only patients older than 16 years of age who were receiving caspofungin for treatment of proven or probable IA and who were not currently participating in another Merck sponsored clinical trial for invasive fungal infections were eligible. Investigators identified eligible patients based on their diagnosis of aspergillus infection and categorized their infection based on their clinical judgment and local practice standards. The sponsors of the study provided guidance to investigators for proven or probable Aspergillus infection diagnosis according to the 2002 European Organisation for Research and Treatment of Cancer/Mycoses Study Group (EORTC/MSG) criteria [11]. Patients with radiologic signs highly suggestive of aspergillosis were eligible for inclusion. There were no protocol-defined exclusion criteria.

\section{Registry Design}

Given the observational nature of this study, no investigational or approved medication was provided to participating centers. All patients were treated according to the standard of care at the participating center. Data were collected between April 2006 and September 2007 across 23 sites in 11 countries including Australia, Belgium, Brazil, Germany, Greece, Jordan, Korea, Russia, Singapore, Slovenia, and Taiwan.

Response was assessed by the investigators at the end of caspofungin therapy (EOCT) using standard definitions [11]. A complete or partial response at EOCT was considered a favorable response; stable disease, failure (progression of disease), or death from any cause was considered an unfavorable response. Complete response required resolution of all attributable clinical signs and symptoms and complete resolution of radiographic or bronchoscopic abnormalities. Partial response required clinically meaningful improvement in all attributable clinical signs and symptoms, a significant improvement of radiographic (at least 50\%) or bronchoscopic abnormalities, and included persistence of radiographic sequelae regardless of the overall level of clinical or radiographic improvement. Stable disease included no improvement of attributable clinical signs or symptoms and no improvement of radiographic or bronchoscopic abnormalities. Failure included deterioration in attributable clinical or radiographic abnormalities that necessitated alternative antifungal therapy or resulted in death.

The safety evaluations required all investigators to report serious and non-serious clinical or laboratory adverse event that was related to the administration of caspofungin. Serious adverse events were those resulting in death or incapacity, were life-threatening, required initial or prolonged hospitalization, and included birth defects, cancer, and those deemed to be serious by medical judgment. An independent expert review panel consisting of two experts (Dr. J. Maertens and Prof. C. Viscoli) reviewed the analysis of the data for accuracy and completeness.

\section{Statistical Analysis}

Patient demographics and baseline patient characteristics were summarized. Patients were categorized according to whether they received caspofungin as monotherapy or whether azoles or polyenes were administered in combination with caspofungin. Summary statistics stratified by monotherapy and combination therapy were also provided for first-line versus secondline use of caspofungin. Caspofungin effectiveness and safety were assessed by the number and percentage of patients with a favorable response at the EOCT and adverse event reports; 95\% confidence intervals were calculated for effectiveness data. Effectiveness was also evaluated for various patient sub-groups including proven vs probable disease; caspofungin monotherapy vs combination therapy; caspofungin as first-line therapy vs salvage therapy; neutropenic status at caspofungin initiation $(<500$ cells $/ \mu \mathrm{L}$ vs $\geq 500$ cells $/ \mu \mathrm{L})$; and risk factors at baseline. Additional summary statistics were provided for length of hospital stay (LOS), survival at 7 days after EOCT, and microbiology.

\section{Results}

A total of 103 patients with proven $(\mathrm{n}=31 ; 30.1 \%)$ or probable ( $n=72 ; 69.9 \%)$ IA was enrolled in the registry. The mean age of the population was 50.4 years and the male-to-female ratio was 2:1 (Table 1). Two patients (2\%) were excluded from the effectiveness analysis because they were still on study therapy at the time the database was closed and therefore were not assessed. 
Table 1 Patient demographics and baseline characteristics

\begin{tabular}{|c|c|c|c|}
\hline Variable & $\begin{array}{l}\text { Monotherapy } \\
(\mathrm{N}=85)\end{array}$ & $\begin{array}{c}\text { Combination therapy } \\
(\mathrm{N}=18)\end{array}$ & $\begin{array}{c}\text { Overall } \\
(\mathrm{N}=103)\end{array}$ \\
\hline \multicolumn{4}{|l|}{ Gender, n (\%) } \\
\hline Male & $55(64.7 \%)$ & $11(61.1 \%)$ & $66(64.1 \%)$ \\
\hline Female & $30(35.3 \%)$ & 7 (38.9\%) & $37(35.9 \%)$ \\
\hline Mean age (years) (mean $\pm S D^{a}$ ) & $49.8 \pm 15.94$ & $53.4 \pm 16.94$ & $50.4 \pm 16.09$ \\
\hline \multicolumn{4}{|l|}{ Race, n (\%) } \\
\hline White & $49(57.6 \%)$ & $15(83.3 \%)$ & $64(62.1 \%)$ \\
\hline Asian & $36(42.4 \%)$ & $3(16.7 \%)$ & 39 (37.9\%) \\
\hline \multicolumn{4}{|l|}{ Country, n (\%) } \\
\hline Germany & $36(42.4 \%)$ & $6(33.3 \%)$ & $42(40.8 \%)$ \\
\hline Korea & $28(32.9 \%)$ & $3(16.7 \%)$ & $31(30.1 \%)$ \\
\hline Russia & $8(9.4 \%)$ & 0 & $8(7.8 \%)$ \\
\hline Taiwan & $6(7.1 \%)$ & 0 & $6(5.8 \%)$ \\
\hline Greece & 0 & $5(27.8 \%)$ & $5(4.8 \%)$ \\
\hline Other & $7(8.2 \%)$ & $4(22.2 \%)$ & $11(10.7 \%)$ \\
\hline Mean APACHE II score (mean \pm SD) & $18.3 \pm 8.26(n=11)$ & $18.3 \pm 2.52(n=3)$ & $18.3 \pm 7.31(n=14)$ \\
\hline SOFA score $($ mean \pm SD) & $8.8 \pm 4.66(n=5)$ & 0 & $8.8 \pm 4.66(n=5)$ \\
\hline \multicolumn{4}{|l|}{ Site of Infection, n (\%) } \\
\hline Blood & $8(9.4 \%)$ & 0 & $8(7.8 \%)$ \\
\hline Lung & $72(84.7 \%)$ & $15(83.3 \%)$ & 87 (84.5\%) \\
\hline Bone/joint & $1(1.2 \%)$ & 0 & $1(1.0 \%)$ \\
\hline Sinus & 0 & $1(5.6 \%)$ & $1(1.0 \%)$ \\
\hline Liver/spleen & 0 & $1(5.6 \%)$ & $1(1.0 \%)$ \\
\hline Other & $2(2.4 \%)$ & 0 & $2(1.9 \%)$ \\
\hline Multiple & $2(2.4 \%)$ & $1(5.6 \%)$ & $3(2.9 \%)$ \\
\hline \multicolumn{4}{|l|}{$\begin{array}{l}\text { Neutropenic at time of initiation of } \\
\text { caspofungin treatment, } \mathrm{n}(\%)\end{array}$} \\
\hline$<500$ cells/ $/ \mathrm{L}$ & $53(62.4 \%)$ & $6(33.3 \%)$ & $59(57.3 \%)$ \\
\hline 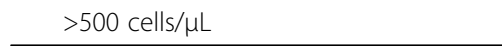 & $32(37.6 \%)$ & $12(66.7 \%)$ & $44(42.7 \%)$ \\
\hline $\begin{array}{l}\text { Number of risk factors per patient } \\
\text { (mean } \pm \text { SD) }\end{array}$ & $5.4 \pm 1.76$ & $4.8 \pm 1.86$ & $5.3 \pm 1.78$ \\
\hline \multicolumn{4}{|c|}{ Risk factors and underlying medical conditions ${ }^{\mathrm{b}}, \mathrm{n}(\%)$} \\
\hline Active malignancy & $70(82.4 \%)$ & $9(50 \%)$ & $79(76.7 \%)$ \\
\hline Immunosuppressive medication & $64(75.3 \%)$ & $12(66.7 \%)$ & $76(73.8 \%)$ \\
\hline Neutropenia at time of hospitalization & $59(69.4 \%)$ & 7 (38.9\%) & $66(64.1 \%)$ \\
\hline Allogeneic HSCT & $18(21.2 \%)$ & $5(27.8 \%)$ & $23(22.3 \%)$ \\
\hline Prior fungal colonization & $16(18.8 \%)$ & $2(11.1 \%)$ & $18(17.5 \%)$ \\
\hline Diabetes mellitus & $11(12.9 \%)$ & $4(22.2 \%)$ & $15(14.6 \%)$ \\
\hline Organ transplantation & $5(5.9 \%)$ & $4(22.2 \%)$ & $9(8.7 \%)$ \\
\hline Autologous HSCT & $5(5.9 \%)$ & 0 & $5(4.9 \%)$ \\
\hline AIDS/HIV infection & $3(3.5 \%)$ & 0 & $3(2.9 \%)$ \\
\hline
\end{tabular}

${ }^{\mathrm{a}} \mathrm{SD}=$ standard deviation

${ }^{b}$ Frequencies of different risk factors and underlying medical conditions are not mutually exclusive.

Pulmonary IA was the most frequent manifestation of infection, accounting for $84.5 \%$ of cases $(\mathrm{n}=87)$, followed by blood infection (fungemia) $(7.8 \%, \mathrm{n}=8)$. Other sites of infection included bone/joint, sinus, liver/ spleen, external ear and trachea (one case each). Three patients had disseminated infection (2.9\%). The main predisposing conditions included malignancy $(76.7 \%)$ and allogeneic HSCT (22.3\%), followed by solid organ transplantation (8.7\%), autologous HSCT (4.9\%), and HIV/AIDS (2.9\%). Many patients presented with multiple predisposing factors with a mean number of 5.3 $(\mathrm{SD}=1.78)$ risk factors present per patient; $57 \%$ of patients were neutropenic ( $\mathrm{ANC}<500$ cells $/ \mu \mathrm{L})$ at the start of caspofungin treatment. 
Table 2 Patients receiving caspofungin treatment

\begin{tabular}{|c|c|c|c|}
\hline Indication for IV caspofungin therapy & $\begin{array}{l}\text { Monotherapy } \\
(\mathrm{N}=85) \text { n (\%) }\end{array}$ & $\begin{array}{c}\text { Combination } \\
\text { therapy } \\
(\mathrm{N}=18) \mathrm{n}(\%)\end{array}$ & $\begin{array}{c}\text { Overall } \\
(\mathrm{N}=103) \mathrm{n}(\%)\end{array}$ \\
\hline Caspofungin first-line therapy & $15(17.6)$ & $5(27.8)$ & $20(19.4)$ \\
\hline Caspofungin salvage therapy & $70(82.4)$ & $13(72.2)$ & $83(80.6)$ \\
\hline Clinical refractory to first-line antifungal & $48(68.6)$ & $10(76.9)$ & $58(69.0)$ \\
\hline Microbiological refractory to first-line antifungal & $6(8.6)$ & $1(7.7)$ & $7(8.4)$ \\
\hline \multicolumn{4}{|l|}{ Toxicity } \\
\hline - Nephrotoxicity to first-line antifungal & $2(2.9)$ & $1(7.7)$ & $3(3.6)$ \\
\hline - Other toxicity & $6(8.6)$ & 0 & $6(7.2)$ \\
\hline Other* & $7(10.0)$ & $1(7.7)$ & $8(9.6)$ \\
\hline Not reported & $1(1.4)$ & 0 & $1(1.2)$ \\
\hline
\end{tabular}

*Other reasons include: Severe disease requiring combination therapy, prophylaxis, elevated Aspergillus antigen, empirical therapy, elevated creatinine

The mean duration of caspofungin therapy was $18.9 \pm$ 21.4 days (range 1-40.3 days). The mean daily dose of caspofungin was $49.3 \pm 6.97 \mathrm{mg}$. In 42 patients, no attempt was made to have a culture-based diagnosis. Of the 61 patients in which culture examination was performed, 34 tested negative, although these patients were identified as having proven/probable IA by the investigator, according to the other criteria (such as positive histopathological evidence), as defined in the EORTC/ MSG guidelines. In culture-positive cases $(\mathrm{n}=27$, 26.2\%), Aspergillus fumigatus was the most frequently isolated species ( $\mathrm{n}=10)$, followed by A. flavus $(\mathrm{n}=3) ; 9$ cases yielded a positive culture for Aspergillus but did not have the species identified. Supportive microbiological data were missing in 5 cases; diagnosis in these cases was based on the investigator's judgment. The majority of patients received caspofungin as salvage therapy $(83 / 103=80.6 \%)($ Table 2$)$. Seventy of these patients received monotherapy and 13 patients received caspofungin-containing combination treatment (usually with voriconazole). The main reasons for switching to salvage therapy included clinical failure of the first-line therapy (69\%), microbiological documentation of persistence of the infection $(8.4 \%)$, or toxicity associated with the firstline therapy $(10.8 \%)$. Antifungals administered prior to switching to caspofungin were amphotericin B deoxycholate (30.1\%), fluconazole (29.1\%), itraconazole (18.4\%), voriconazole $(12.6 \%)$ or a lipid-based formulation of amphotericin B (11.7\%). Twenty patients (19.4\%) received caspofungin as first-line monotherapy $(\mathrm{n}=15)$ or combination therapy $(\mathrm{n}=5)$.

\section{Response at EOCT}

An overall favorable response rate of 56.4\% (57/101) was observed in this study population. Patients receiving caspofungin monotherapy and combination therapy had response rates of $56.3 \%(9 / 16)$ and $56.5 \%(48 / 85)$, respectively (Table 3 ). Those receiving first-line therapy had a slightly higher response rate (60.0\%) than those receiving salvage therapy (55.6\%). Caspofungin first-line monotherapy was associated with a $60.0 \%$ (9/15) favorable response. Favorable response rates in clinically

Table 3 Favorable response (complete plus partial) by patient subgroup $(\mathrm{N}=101)$

\begin{tabular}{lc}
\hline Variable & $\begin{array}{c}\text { Favorable response } \\
\%(\mathbf{n} / \mathbf{N})[95 \% \mathbf{C l}]\end{array}$ \\
\hline Overall & $56.4(57 / 101)[46.7 ; 66.1]$ \\
\hline Probable aspergillosis & $56.3(40 / 71)[44.0 ; 68.1]$ \\
Proven aspergillosis & $56.7(17 / 30)[37.4 ; 74.5]$ \\
\hline Combination therapy & $56.3(9 / 16)[29.9 ; 80.2]$ \\
$\quad$ First line & $60.0(3 / 5)[14.6 ; 94.7]$ \\
$\quad$ Second Line & $54.6(6 / 11)[23.4 ; 83.3]$ \\
Monotherapy & $56.5(48 / 85)[45.3 ; 67.2]$ \\
$\quad$ First line & $60.0(9 / 15)[32.3 ; 83.7]$ \\
$\quad$ Second Line & $55.7(39 / 70)[43.3 ; 67.6]$ \\
\hline First-line therapy & $60.0(12 / 20)[36.1 ; 80.9]$ \\
Salvage therapy & $55.6(45 / 81)[44.1 ; 66.6]$ \\
\hline Culture examination performed $\quad 62.3(38 / 61)[50.1 ; 74.5]$ \\
$\quad$ Positive & $55.6(15 / 27)[36.9 ; 76.6]$ \\
$\quad$ Negative & $67.6(23 / 34)[49.5 ; 82.6]$ \\
\hline
\end{tabular}

Neutropenic status at start of caspofungin therapy

\begin{tabular}{lc} 
ANC $<500$ cells/ $\mu \mathrm{L}$ & $52.5(31 / 59)[39.1 ; 65.7]$ \\
ANC $>=500$ cells/ $\mu \mathrm{L}$ & $61.9(26 / 42)[45.6 ; 76.4]$ \\
\hline Risk factors & \\
Active malignancy & $51.9(41 / 79)[40.4 ; 63.3]$ \\
AIDS/HIV infection & $66.7(2 / 3)[9.4 ; 99.2]$ \\
Bone marrow/stem cell transplantation & $53.6(15 / 28)[33.9 ; 72.5]$ \\
• Autologous HSCT & $40.0(2 / 5)[5.3 ; 85.3]$ \\
• Allogeneic HSCT & $56.5(13 / 23)[34.5 ; 76.8]$ \\
Diabetes mellitus & $57.1(8 / 14)[28.9 ; 82.3]$ \\
Immunosuppressive medication & $60.8(45 / 74)[48.8 ; 72.0]$ \\
Neutropenia at the time of & $53.0(35 / 66)[40.3 ; 65.4]$ \\
hospitalization & \\
Prior fungal colonization & $61.1(11 / 18)[35.7 ; 82.7]$ \\
Organ transplantation & $75.0(6 / 8)[34.9 ; 96.8]$ \\
\hline
\end{tabular}

Response evaluated in $\mathrm{N}=101$ patients. 
relevant subgroups included a $51.9 \%$ response rate in patients with active malignancy, $56.5 \%$ in those with allogeneic HSCT and $53.0 \%$ in patients with neutropenia (Table 3).

\section{Outcomes}

The overall LOS was 59.9 days. Increased LOS due to adverse events was observed in 1 patient $(1.0 \%)$, and increased LOS due to treatment failure with caspofungin was observed in 7 patients $(6.8 \%)$. Survival rates of $74.1 \%(63 / 85)$ were observed at 7 days after the end of caspofungin monotherapy and $62.5 \%(10 / 16)$ at 7 days after the end of combination therapy.

\section{Safety}

No patient discontinued caspofungin therapy due to drug interactions between caspofungin and other antifungal drug. According to the investigator, 3/103 patients (2.9\%) experienced a serious drug-related clinical adverse event (bronchopneumonia, respiratory failure, skin reaction), including two patients $(1.9 \%)$ who discontinued caspofungin therapy (Table 4). One patient (1.0\%) developed a serious laboratory adverse event (hyperbilirubinemia) that resulted in discontinuation of caspofungin therapy. Twenty-three patients $(27.1 \%)$ in the monotherapy and 5 patients $(27.8 \%)$ in the combination therapy group died. No reports were received from investigators attributing any death to caspofungin therapy.

\section{Discussion}

Caspofungin regimens provided high favorable response and survival rates in this observational registry documenting real world findings. An overall favorable response rate of $56.3 \%$ was observed in patients with proven or probable aspergillosis treated with caspofungin monotherapy or combination therapy. A total of 20/ 101 patients received caspofungin as first-line therapy (as part of a combination regimen in 5 patients), which was associated with a $60.0 \%$ favorable response. Favorable responses $>50 \%$ were seen in high risk patient subgroups, including those with a malignancy (51.9\%), with allogeneic HSCT (56.5\%) or neutropenia at the time of hospitalization $(53.0 \%)$. There was a $73 \%$ survival at 7 days after EOCT. A low rate of drug-related serious adverse events and drug-related adverse events leading to discontinuation was observed, with no differences noted between the safety profiles of caspofungin monotherapy and combination therapy.

The high rates of favorable response observed in this registry were impressive considering the severity of illness in the patient population. The majority of patients in this study $(81.6 \%)$ required salvage therapy due to prior treatment failure or toxicity. The patients included in this registry were very ill at baseline, as evidenced by the high proportion of patients with neutropenia (57.3\%) and active malignancies (76.7\%). Additionally, some patients received caspofungin after failing amphotericin B therapy due to lack of efficacy or intolerance. Therefore, it should be considered that long-term persistence of relevant amphotericin $B$ concentration in organ tissue may have led to effectiveness not solely attributable to caspofungin. It is also worth noting that survival was lower for patients who received combination therapy as this group was likely composed of a sicker population with a greater disease burden in comparison to those selected for monotherapy.

The favorable effectiveness and survival results presented in this registry were consistent with the cumulative published literature on caspofungin. The registry data confirm the previously reported efficacy of caspofungin monotherapy [6] and combination therapy [7] in clinical trials. The favorable response rates and survival rates in our study reflected trends observed with liposomal amphotericin B and voriconazole when used as first-line therapy, although there was a difference in the time to follow up between this and the published studies [4]. The median duration of therapy was also shorter in this study than in clinical studies of caspofungin therapy (median, 25 days, range, 1-196 days; median, 28 days; range, 1-162 days) [6,7]. In a double-blind trial of patients $(n=201)$ with invasive mold infections, $97 \%$ of whom had IA, $50 \%$ of patients achieved a favorable response with liposomal amphotericin B and $72 \%$ survived to at least 12 weeks [5]. A randomized, unblinded study of patients $(\mathrm{n}=144)$ with IA found that $52.8 \%$ of patients who received voriconazole had a favorable

Table 4 Adverse events

\begin{tabular}{lcc}
\hline Type of Adverse Events & Clinical Adverse Events* & $\begin{array}{c}\text { Laboratory Adverse Events** } \\
\mathbf{N}=\mathbf{1 0 3} \\
\mathbf{n}(\%)\end{array}$ \\
\hline Any adverse events & $\mathbf{n}(\%)$ & $8(7.8)$ \\
Serious adverse events & $4(3.9)$ & $1(0.9)$ \\
Serious adverse events leading to discontinuation & $3(2.9)$ & $1(0.9)$ \\
\hline
\end{tabular}

* Clinical adverse events include: bronchopneumonia; skin reaction; respiratory failure; and abdominal pain

**Laboratory adverse events include: increase in aspartate aminotransferase, alanine aminotransferase, blood alkaline phosphatase, blood bilirubin, gamma glutamyltransferase; leukopenia; hyperbilirubinemia; and hypokalemia. 
response, compared with only $31.6 \%$ who received amphotericin B; 12 -week survival rates of $70.8 \%$ and $57.9 \%$ were achieved in the voriconazole and amphotericin B groups, respectively [4]. A prospective database study of 85 patients with allogeneic HSCT, 24 with graft versus host disease, found an overall response rate of $31 \%$ with amphotericin B lipid complex as first- or second-line therapy [12].

The present registry had several inherent limitations. As an observational registry documenting real world use of caspofungin in proven and probable IA, no comparator arm was available and no superiority or non-inferiority could be determined. In addition, the low numbers of patients in some of the categories may have skewed the results. While deaths occurring during the observation period were determined to be unrelated to caspofungin, the reasons for death were not collected with the study data. Diagnosis of proven and probable IA was based on investigators' clinical judgment and local practice standards using the EORTC/MSG criteria as a guide. All sites were not mandated to provide comprehensive diagnostic information and treatment practices for IA, which may have varied between countries and regions. This could have resulted in misclassification of disease. Cases with changes highly suggestive of IA on radiology were eligible for study inclusion as probable IA, whereas EORTC/MSG criteria would categorize these cases as possible IA. Therefore, possible cases could have been included as probable cases resulting in a bias away from null and hence, an overestimation of response rates. As an observational study, reports of fungemia were accepted as recorded by investigators and all patients with proven or probable IA were included. Many patients with A. fungemia have a low performance score and exhibit co-morbidities which excludes them from clinical studies, but not from registries; therefore, the number of fungemia cases in our study may have been greater than expected. Finally, there was a limited follow-up period after caspofungin therapy ended.

\section{Conclusions}

In this observational registry of daily clinical practice, antifungal regimens in which caspofungin was used as monotherapy or combination therapy, and as either first-line or second-line therapy, provided effective treatment in severely ill patients with proven or probable IA. Caspofungin was both effective and well tolerated among high-risk patient groups such as those with neutropenia and active malignancies.

\section{List of Abbreviations}

EOCT: End of caspofungin therapy; EORTC/MSG: European Organisation for Research and Treatment of
Cancer/Mycoses Study Group; HSCT: Hematopoietic stem cell transplantation; IA: Invasive aspergillosis; SD: Standard deviation.

\section{Acknowledgements}

Study design: Prospective observational registry. This Registry was sponsored by Merck \& Co., Inc. Partial content was presented at the 18th European Congress of Clinical Microbiology and Infectious Diseases (ECCMID) meeting in Barcelona, Spain, from April 19-22, 2008. Writing assistance was provided by Wendy Horn, PhD, with funding from Merck \& Co., Inc. We acknowledge Syreon Corp. for data management. We gratefully acknowledge the CAN-DO(IA) study team for contributing patients: Dr. Sharon Chen from Australia; Dr. Dominik Selleslag from Belgium; Dr. Flavio de Queiroz Telles Filho from Brazil; Dr. Mathias Pletz from Germany; Prof. Panagiota Matsouka, Dr. Vasilios Seitanidis, Dr. George L. Petrikkos from Greece; Dr. Mustafa M Saad from Jordan; Dr. Kyong Ran Pec, Dr. Sang-Ho Choi, Dr. Jun-Ho Jang, Dr. Jun-Yong Choi from Korea; Dr. Galina Kliasova, Dr. Natalia V. Dimitrieva, Dr. Tatiana S. Konstantinova from Russia; Dr. Gee Chuan Wong from Singapore; Dr. Tatjana Lejko Zupanc from Slovania; Dr. Yee-Chun Chen, Dr. Yung-Ching Liu from Taiwan.

\section{Author details}

${ }^{1}$ UZ Gasthuisberg, Leuven, Belgium. ${ }^{2}$ Universitätsklinikum Heidelberg, Heidelberg, Germany. ${ }^{3}$ Department of Internal Medicine, The Catholic University of Korea College of Medicine, Seoul, Korea. ${ }^{4}$ Klinikum Oldenburg gGmbH, Oldenburg, Germany. ${ }^{5}$ ID Consultant Services, Ambler, PA, USA. 6UMDNJ- Robert Wood Johnson Medical School, Department of Family Medicine- Research Division, Somerset, NJ, USA. ${ }^{7}$ Merck \& Co., Inc. Whitehouse Station, NJ, USA. ${ }^{8}$ San Martino University Hospital, Genova, Italy.

\section{Authors' contributions}

SC and MS had full access to all of the data in the study and take responsibility for the integrity of the data and the accuracy of the data analysis. JM, MS, MS, and CV conceived the study and participated in its design. JM, GE, WSS, DR, and MS were responsible for acquisition of data. $\mathrm{JM}, \mathrm{MS}, \mathrm{SC}, \mathrm{MS}$, and $\mathrm{CV}$ were responsible for interpretation of data. MS and SC participated in drafting the manuscript. All authors participated in critical revision of the manuscript for important intellectual content. MS was responsible for study supervision. All authors read and approved the final manuscript. CAN-DO(IA) study team was responsible for contributing patients in the study.

\section{Competing interests}

J. Maertens has received research grants from Merck/MSD and Pfizer; is a consultant to Astellas, Bio-Rad, Merck/MSD, Nektar, Pfizer, Schering-Plough, F2G, and Zeneus/Cephalon; and has served at the speakers' bureau of Astellas, Bio-Rad, Merck/MSD, Pfizer, Schering-Plough, and Zeneus/Cephalon. $\mathrm{G}$. Egerer has received research funding from MSD SHARP \& DOHME GmbH and Schering-Plough; has been a member of the speaker's bureau of MSD, SHARP \& DOHME GmbH and Schering-Plough; and has received travel grants from MSD SHARP \& DOHME GmbH and Schering-Plough.

M Stek was an employee of Merck \& Co. Inc. (manufacturer of caspofungin) when the study was designed and since retirement from the company has served as a consultant to Merck \& Co., Inc.

S. Chandwani is currently an employee of UMDNJ. At the time of the study she was participating in a joint fellowship between Merck \& Co., Inc. and Rutgers University.

M. Shivaprakash is an employee of Merck \& Co., Inc, which manufactures caspofungin.

C. Viscoli has served on speakers' bureaus for Merck, Pfizer, Schering-Plough, and Gilead; has served on advisory boards for Merck, Pfizer, Schering-Plough, Gilead, and Astellas; and has received research grants from Gilead, Abbott, Boeheringer-Ingelheim, and Pfizer. P.G.P. has received research grants from Merck, Pfizer, SPRI, and Astellas; has served on speakers' bureaus for Merck, Pfizer, and Astellas; and has been an ad hoc advisor for Merck, SPRI, Pfizer, Astellas, Novartis, and Eisi

Received: 27 May 2009 Accepted: 22 June 2010 Published: 22 June 2010 


\section{References}

1. Walsh TJ, Anaissie EJ, Denning DW, Herbrecht R, Kontoyiannis DP, Marr KA Morrison VA, Segal BH, Steinbach WJ, Stevens DA, van Burik JA, Wingard JR, Patterson TF: Treatment of aspergillosis: clinical practice guidelines of the Infectious Diseases Society of America. Clin Infect Dis 2008, 46(3):327-360.

2. Lin SJ, Schranz J, Teutsch SM: Aspergillosis case-fatality rate: systematic review of the literature. Clin Infect Dis 2001, 32(3):358-366.

3. Kontoyiannis DP, Bodey GP: Invasive aspergillosis in 2002: an update. Eur J Clin Microbiol Infect Dis 2002, 21(3):161-172.

4. Herbrecht R, Denning DW, Patterson TF, Bennett JE, Greene RE, Oestmann JW, Kern WW, Marr KA, Ribaud P, Lortholary O, Sylvester R, Rubin RH, Wingard JR, Stark P, Durand C, Caillot D, Thiel E, Chandrasekar PH, Hodges MR, Schlamm HT, Troke PF, de Pauw B: Voriconazole versus amphotericin $B$ for primary therapy of invasive aspergillosis. N Engl J Med 2002, 347(6):408-415.

5. Cornely OA, Maertens J, Bresnik M, Ebrahimi R, Ullmann AJ, Bouza E, Heussel CP, Lortholary O, Rieger C, Boehme A, Aoun M, Horst HA, Thiebaut A, Ruhnke M, Reichert D, Vianelli N, Krause SW, Olavarria E, Herbrecht R: Liposomal amphotericin B as initial therapy for invasive mold infection: a randomized trial comparing a high-loading dose regimen with standard dosing (AmBiLoad trial). Clin Infect Dis 2007, 44(10):1289-1297.

6. Maertens J, Raad I, Petrikkos G, Boogaerts M, Selleslag D, Petersen FB, Sable CA, Kartsonis NA, Ngai A, Taylor A, Patterson TF, Denning DW, Walsh TJ: Efficacy and safety of caspofungin for treatment of invasive aspergillosis in patients refractory to or intolerant of conventional antifungal therapy. Clin Infect Dis 2004, 39(11):1563-1571.

7. Maertens J, Glasmacher A, Herbrecht R, Thiebaut A, Cordonnier C, Segal BH, Killar J, Taylor A, Kartsonis N, Patterson TF, Aoun M, Caillot D, Sable C: Multicenter, noncomparative study of caspofungin in combination with other antifungals as salvage therapy in adults with invasive aspergillosis. Cancer 2006, 107(12):2888-2897.

8. Kartsonis NA, Nielsen J, Douglas CM: Caspofungin: the first in a new class of antifungal agents. Drug Resist Updat 2003, 6(4):197-218

9. Glasmacher A, Cornely OA, Orlopp K, Reuter S, Blaschke S, Eichel M, Silling $G$, Simons B, Egerer $G$, Siemann M, Florek M, Schnitzler R, Ebeling $P$, Ritter J, Reinel H, Schutt P, Fischer H, Hahn C, Just-Nuebling G: Caspofungin treatment in severely ill, immunocompromised patients: a case-documentation study of 118 patients. J Antimicrob Chemother 2006, 57(1):127-134.

10. International conference on harmonisation of technical requirements for registration of pharmaceuticals for human use. Guideline for good clinical practice. [http://www.ich.org/LOB/media/MEDIA482.pdf].

11. Ascioglu S, Rex JH, de Pauw B, Bennett JE, Bille J, Crokaert F, Denning DW Donnelly JP, Edwards JE, Erjavec Z, Fiere D, Lortholary O, Maertens J, Meis JF, Patterson TF, Ritter J, Selleslag D, Shah PM, Stevens DA, Walsh TJ: Defining opportunistic invasive fungal infections in immunocompromised patients with cancer and hematopoietic stem cell transplants: an international consensus. Clin Infect Dis 2002, 34(1):7-14.

12. Ito Jl, Chandrasekar PH, Hooshmand-Rad R: Effectiveness of amphotericin $B$ lipid complex (ABLC) treatment in allogeneic hematopoietic cell transplant $(\mathrm{HCT})$ recipients with invasive aspergillosis (IA). Bone Marrow Transplant 2005, 36(10):873-877.

\section{Pre-publication history}

The pre-publication history for this paper can be accessed here: http://www.biomedcentral.com/1471-2334/10/182/prepub

doi:10.1186/1471-2334-10-182

Cite this article as: Maertens et al:: Caspofungin Use in Daily Clinical Practice for Treatment of Invasive Aspergillosis: Results of a Prospective Observational Registry. BMC Infectious Diseases 2010 10:182.

\section{Submit your next manuscript to BioMed Central and take full advantage of:}

- Convenient online submission

- Thorough peer review

- No space constraints or color figure charges

- Immediate publication on acceptance

- Inclusion in PubMed, CAS, Scopus and Google Scholar

- Research which is freely available for redistribution

Submit your manuscript at www.biomedcentral.com/submit
Biomed Central 